\title{
The Perception of Daily Temperatures as Evidence of Global Warming $\mathscr{O}$
}

\author{
STEPHEN B. BROOMELL \\ Department of Social and Decision Sciences, Carnegie Mellon University, Pittsburgh, Pennsylvania \\ JON-FRANCIS WINKLES \\ Heinz College, Carnegie Mellon University, Pittsburgh, Pennsylvania \\ PATRICK BODILLY KANE \\ Department of Social and Decision Sciences, Carnegie Mellon University, Pittsburgh, Pennsylvania
}

(Manuscript received 5 January 2017, in final form 31 March 2017)

\begin{abstract}
Unlike the scientific definition of global warming $(\mathrm{GW})$, public discussion often links the existence of GW to daily temperatures rather than long-term averages. Previous research found that daily weather is perceived as personal experiences with GW. Additionally, prior beliefs about GW can affect interpretations of such experiences as evidence for the existence of GW. However, previous studies demonstrating that beliefs affect interpretations of experiences were based on correlational designs-limiting causal inferences—and relied only on self-reports of remembered personal experiences instead of direct interpretations of weather. The authors present the first randomized experiment investigating how people interpret daily temperatures in terms of the evidence that it provides about GW, clarifying the psychological causes for different interpretations of the same experiences across individuals. They test the influence of knowledge about (and beliefs in) GW on the interpretation of daily temperatures across two framing conditions labeled weather (interpreting a temperature as abnormal weather) and climate (interpreting a temperature as evidence of $\mathrm{GW}$ ). The authors use signal detection theory to measure the decision-maker's (a) ability to discriminate between temperatures, called sensitivity, and (b) threshold for describing a temperature as abnormal, called the decision threshold. The results replicate previous research finding a motivational distortion in interpreting temperatures as evidence of GW and further find belief-consistent distortions in decision thresholds while observing no measurable change in sensitivity. In other words, people know when temperatures are abnormally hot, but classify ambiguous events (i.e., less extreme abnormalities) differently based on their beliefs in GW.
\end{abstract}

\section{Introduction}

Scientists predict that global warming $(\mathrm{GW})$ will lead to changes in the climate that can be characterized in part by an increase in the occurrence of certain weather events (Trenberth 2012), with the type and severity of such events varying by location (IPCC 2014). These events include (but are not limited to) rising average temperature, rising sea levels, and increases in the

Supplemental information related to this paper is available at the Journals Online website: http://dx.doi.org/10.1175/ WCAS-D-17-0003.s1.

Corresponding author: Stephen B. Broomell, broomell@gmail. com frequency of severe weather. However, no single weather event can be unambiguously attributed to GW because climate change is measured across centuries using large-scale averages (e.g., annual mean global temperature).

In contrast to the scientific definition of GW, psychological studies have found that the public is willing to make inferences from local hot spells to $\mathrm{GW}$, called the local warming effect ( $\mathrm{Li}$ et al. 2011; McCright et al. 2014; Risen and Critcher 2011; Zaval et al. 2014). Specifically, the public often links the existence of GW to daily temperatures (and physically proximate experiences) rather than long-term, large-scale averages. This effect is a special case of attribute substitution (Kahneman and Frederick 2002), a heuristic judgment process that bases inferences on easily observable attributes (in this case, 
daily temperature) in lieu of hard-to-observe ones (i.e., global average trends). This psychological process can lead to problems for reaching consensus about GW because (a) different locations will have different experiences (Melillo et al. 2014) and (b) members of the public may have different interpretations of the same experiences in terms of the evidence it provides for $\mathrm{GW}$ (Goebbert et al. 2012; Howe and Leiserowitz 2013; Myers et al. 2013).

These erroneous inferences and judgments based on daily weather can therefore undermine warranted confidence in GW (e.g., interpreting cold spells as contrary evidence) or overinflate confidence beyond that warranted by scientific evidence (e.g., interpreting all heat waves as being caused only by GW). This process can also lead the public and experts to misunderstand and perhaps mistrust one another, contributing to polarized positions on GW (Howe and Leiserowitz 2013; Weber and Stern 2011). Since personal experiences are a strong motivator for concern for $\mathrm{GW}$ and willingness to take actions mitigating its effects (Zaval et al. 2014, Broomell et al. 2015; Weber 2006; Weber 2013), they can differentially motivate subgroups with certain types of experiences to act more than others (Spence et al. 2011).

We build upon this body of research by focusing on the cognitive mechanisms through which beliefs about GW affect interpretations of everyday weather. Individuals with different beliefs may have diverging interpretations of temperatures because of (a) differences in knowledge and experience with daily temperatures (Weber and Stern 2011) or (b) different interpretations of what constitutes an abnormal temperature (Kahan et al. 2012). Understanding the primary driver of why different members of the public perceive the same experiences differently can point toward effective interventions for building consensus about the existence of GW (Weber 2013) and intentions to perform mitigating actions (Broomell et al. 2015).

Here, we introduce a novel approach to analyzing judgments of daily temperatures as evidence of GW to investigate sources of bias in perceptions of personal experiences of GW. We seek to understand if people who believe in GW see it everywhere or if people who do not believe in global warming refuse to see it. Both of these situations depend on understanding whether believers and nonbelievers have similar ability to perceive daily temperatures as abnormal. Our goal is to assess the extent to which people perceive daily temperatures as abnormal and whether these perceptions are influenced by a local warming mental model, not to assess laypeople's ability to recognize actual evidence of GW (i.e., increases in global average temperatures). Specifically, we use signal detection theory to measure the extent to which people tend to identify temperatures as abnormal and how their performance on this task relates to beliefs about GW. We compare performance in a condition where participants identify abnormal weather against a condition where participants identify each temperature as evidence of GW to isolate the effect of GW beliefs on interpreting temperatures. We first introduce signal detection theory as our modeling framework for studying judgments of daily temperatures. We then present an experiment that asks participants to judge a collection of daily temperatures that could be observed in their local area. Finally, we discuss the results of the experiment and implications for communicating about GW.

\section{a. Signal detection theory}

We use signal detection theory (SDT; Green and Swets 1988; Macmillan and Creelman 2004; Wickens 2001) to distinguish two pathways of influence: (a) how well people extract information content from their personal experience with local weather, called their sensitivity, and (b) how abnormal an experience must be to constitute evidence of $\mathrm{GW}$, called their decision threshold. SDT studies ask people whether each of many noisy stimuli contains a genuine signal (see, e.g., Farris et al. 2008a,b; Dewitt et al. 2015). We first describe how this experimental paradigm can be applied to understanding how people interpret temperatures in terms of GW and then describe how we implemented and interpreted this framework for our experiment.

Imagine observing temperatures from two different hypothetical Earths: one Earth free of GW where observed temperatures are generated from a stable (unchanging) distribution that represents normal temperatures and a second Earth with GW where observed temperatures are generated from a distribution that is, on average, warmer than normal (for simplicity, assume identical variances). Imagine observing a series of temperatures measured on both hypothetical Earths without knowing the true source of the temperature. The task is to identify which temperatures were measured on the hypothetical Earth with GW (henceforth, Earth GW) and which temperatures were measured on the hypothetical earth without GW (henceforth, Earth Normal). In this example, Earth GW represents the signal and Earth Normal represents noise. Figure 1 depicts a hypothetical signal detection model. Observations from Earth Normal are described by the distribution on the left (with cooler temperatures translating to less evidence on average) and observations from Earth GW are described by the distribution on the right (with warmer temperatures translating to more evidence on average). While very high and very low temperatures can more easily be identified as evidence for 


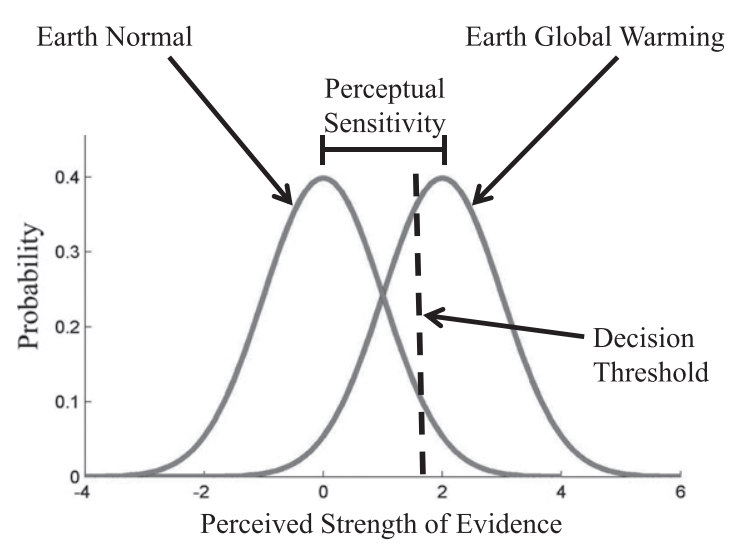

FIG. 1. A depiction of the perceptual sensitivity and decision threshold based on a Gaussian signal detection model. The $x$ axis represents the perceived strength of evidence. The $y$ axis represents the chances of encountering the perceived evidence (which is based on a particular temperature) given the observation was drawn from one of the two hypothetical Earths.

Earth GW and Earth Normal respectively, temperatures in between have some amount of indeterminacy. Signal detection theory models how this indeterminacy is resolved by observing an individual's judgments.

There are two important dimensions of judgment that can be extracted from this type of experimental setup. The first dimension reflects the decision-maker's ability to correctly distinguish temperatures measured on each Earth, and is called sensitivity. Sensitivity is depicted in Fig. 1 as the distance between the centers of the two distributions along the perceived evidence continuum. This distance is a function of the stimuli (i.e., the objective difference between signal and noise) and a function of the observer's ability (i.e., an individual's ability to see the difference). A hypothetical Earth warmed by $20^{\circ} \mathrm{F}$ would lead to easier separation of observed temperatures for perceiving evidence for signal versus noise than a hypothetical Earth warmed by $1^{\circ} \mathrm{F}$. For a given set of stimuli, an individual who is good at distinguishing temperatures has more sensitivity than an individual who is not good a distinguishing temperatures. Therefore, estimates of sensitivity can measure perceptual ability for a given task and reveal if individuals who do not believe in GW generally pay less attention to daily temperatures, and therefore have difficulty recognizing an abnormal temperature.

The second dimension reflects the threshold for giving a particular response, called the decision threshold (or response bias). The decision threshold is measured using the relative frequency of false alarms (identifying a temperature as signal when it is noise) and correct hits (correctly identifying signals), indicating the strength of evidence required for a participant to provide a particular response. In our example, the decision threshold represents how warm an observed temperature needs to be before an experimental participant will classify the observation as being generated by Earth GW. The decision threshold is depicted in Fig. 1 with the vertical dashed line. The threshold can be shifted upward or downward due to factors related to the decision context: (a) the base rate of observing temperatures from a particular hypothetical Earth and (b) the relative value of correct classification versus mistakes. For instance, we may want a decision threshold to be biased in favor of Earth Normal if we know Earth Normal is more likely to be observed. We may also want a decision threshold to be biased in favor of Earth GW if we believe that misclassifying Earth GW as Earth Normal could lead to catastrophic outcomes. Estimates of decision threshold measure the decision strategy for classifying temperatures and can reveal if individuals who do not believe in GW use different decision strategies for judging daily temperatures than individuals who do believe in GW.

Our experiment is very similar to this example with a few subtle differences. The participants in our experiment are making judgments about stimuli that are temperatures in their local area that span the natural distribution of historic observations for that area. We randomly assign participants to one of two different tasks: (a) detection of abnormal weather and (b) detection of GW based on weather. When detecting abnormal weather, we model the signal as an abnormal temperature relative to their local area's distribution of temperatures, and noise as a temperature that fits their area's normal distribution of temperatures. Sensitivity is how well abnormal temperatures are identified. The decision threshold is how extreme a temperature must be in order to be classified as abnormal. When detecting global warming, we model the signal as temperatures that are warmer than average for their local area (representing local warming). Sensitivity is how well warmer than average temperatures are identified. The decision threshold is how warm a temperature must be in order to be classified as GW. We define abnormal temperatures and the mental model for global warming in section $2 \mathrm{~d}$. See the online supplemental material for an extended description of SDT.

\section{b. Research goals}

Previous research has found that belief in GW affects self-reports of personal experiences with weather (Goebbert et al. 2012; Howe and Leiserowitz 2013; Myers et al. 2013) as a special case of motivated cognition in interpreting ambiguous evidence (Nickerson 1998; Kunda 1990; Lewandowsky et al. 2013, Budescu et al. 2012; 
McCright and Dunlap 2011). However, it is unclear if motivation affects overall engagement with weather information and knowledge about normal temperature distributions, thereby affecting their sensitivity, or if motivation affects what information subjects choose to call abnormal, thereby affecting their decision threshold. Since previous studies do not distinguish sensitivity and decision threshold in weather judgments (which SDT allows us to do), they cannot say which motivational mechanism is at work. Additionally, previous studies relied solely on self-reports of remembered personal experiences with $\mathrm{GW}$, which could be biased by memories of personal experiences being selectively stored and recalled as a function of belief.

We present the results of an experiment designed to test how an individual's beliefs about GW affect their interpretation of weather. We also control for additional variables that include age, education, knowledge about causes of GW, and political ideology. Our experiments reveal systematic associations between decision threshold and belief in $\mathrm{GW}$ in interpreting temperatures as evidence of global warming. These associations are not observed in interpreting temperatures as abnormal weather. Taken together, these results suggest a motivational distortion in interpreting temperatures as evidence of GW, with belief in GW distorting the decision threshold, but not sensitivity. We find that people know when temperatures are abnormally hot, but they classify ambiguous events that consist of less extreme abnormalities differently, in accordance with their beliefs in GW.

\section{Methods}

\section{a. Participants}

We determined a target sample size of 200 participants before data collection. We collected 207 responses to an online survey administered to residents of the greater Cincinnati and Pittsburgh metropolitan areas. We recruited participants from Pittsburgh and Cincinnati because these two cities have similar weather but somewhat different politics. Of the total sample, $41 \%$ of responses were from Cincinnati and $71 \%$ of respondents were female. Respondents were collected through the Clearvoice research panel (www.clearvoice.com) between 15 August and 16 September 2014 and were paid the flat rate participation fee offered by Clearvoice. We had 303 people start but not complete the survey. Participation was terminated for those who self-reported that they did not live in the required locations (Cincinnati or Pittsburgh) or failed to correctly respond to an attention check. The full demographic composition of the sample is displayed in Table 1.
TABLE 1. Demographic distribution of survey sample.

\begin{tabular}{lrc}
\hline \multicolumn{1}{c}{ Demographics } & Frequency & Percentage \\
\hline Location & & \\
Cincinnati & 84 & 0.41 \\
Pittsburgh & 123 & 0.59 \\
Gender & & \\
Female & 147 & 0.71 \\
Male & 60 & 0.29 \\
Political party & & \\
Democrat & 80 & 0.39 \\
Independent & 75 & 0.36 \\
Republican & 52 & 0.25 \\
Education & & \\
$\quad$ Some high school & 3 & 0.01 \\
High school & 45 & 0.22 \\
Some college & 66 & 0.32 \\
Bachelor's degree & 66 & 0.32 \\
Graduate degree & 24 & 0.12 \\
Other & 3 & 0.01 \\
\hline
\end{tabular}

\section{b. Design}

To identify the way in which beliefs regarding GW influence individual interpretations of weather, we compared performance between two groups completing an identical task with different framing. In the weather frame, participants were instructed to classify temperatures as either normal (noise) or abnormal (signal), with no mention of GW. In the climate frame, participants were instructed to classify temperatures as either not evidence of GW (noise) or evidence of GW (signal). We also measure each participant's beliefs, knowledge, and ideology related to GW. We predicted that an individual's beliefs about GW would affect their judgments about whether temperatures reflect GW but not affect their judgments about whether the temperatures reflect abnormal weather.

Participants from both Pittsburgh and Cincinnati performed two signal detection style tasks: (a) perception of temperatures, where they classified 32 temperatures drawn from four different months representing the seasons (winter, spring, summer, and fall), and (b) knowledge of causes, where they classified 16 potential causes of GW from a list containing both actual and bogus causes. For each participant, we estimated their sensitivity and decision threshold for both of these tasks. Participants additionally responded to a collection of scales measuring belief in $\mathrm{GW}$ and provided demographic information. We analyzed the dependent variables of sensitivity and threshold in temperature perception as a function of (a) the framing condition (weather or climate), (b) location (Cincinnati or Pittsburgh), (c) participant demographics, (d) participants' sensitivity and threshold in knowledge of causes, and 
(e) participants' responses to six scales measuring beliefs about GW: belief in the existence of $\mathrm{GW}$, belief in personal experiences with GW, pro-environmental worldviews, belief in response-efficacy of cooperation, intentions to act to mitigate the effects of $\mathrm{GW}$, and belief in the free market system.

\section{c. Procedure}

After consenting to partake in the experiment, participants were asked to verify that they were a resident of either Cincinnati or Pittsburgh, and were randomly assigned to either the Weather or Climate condition. Individuals assigned to the Weather condition read the following instructions:

\begin{abstract}
"Weather patterns are highly fluctuating and uncertain for many regions of the country. The local area of [Cincinnati/ Pittsburgh] can experience changes from usual temperatures from time to time. You will be shown a set of temperature readings for [Cincinnati/Pittsburgh]. These readings were taken during different months. Imagine that these temperature readings are recent readings within [Cincinnati/Pittsburgh]. Your task is to tell us your interpretation of these temperature readings as either from a normal day or an abnormal day for the given month. For each temperature you will be asked to rate your confidence in your decision."
\end{abstract}

Individuals assigned to the Climate condition read the following instructions:

"Climatologists have predicted that significant changes will occur in the earth's temperatures and atmosphere in the next 100 years as a result of $\mathrm{CO}_{2}$ emissions. Climate scientists have predicted an increase in extreme weather events and that average global temperatures will increase by roughly 3 degrees Fahrenheit by 2100 . This phenomenon is commonly labeled global warming (or global climate change). Predictions of future changes include an increase in the occurrence of certain types of weather (and disaster) events that occur naturally today, though not all weather events that occur today are consistent with future global warming predictions. Therefore, current weather can either support the predictions of global warming or not support the predictions of global warming.

Weather patterns are highly fluctuating and uncertain for many regions of the country, and the local area of [Cincinnati/Pittsburgh] can experience changes from usual temperatures from time to time. You will be shown a set of temperature readings for [Cincinnati/ Pittsburgh]. Assume these readings represent the temperatures for different months. Imagine that these temperature readings are recent readings within [Cincinnati/ Pittsburgh]. Your task is to tell us your interpretation of these temperature readings as either evidence of global warming or not evidence of global warming for the given month. For each temperature you will be asked to rate your confidence in your decision."

Participants then proceeded to classify 32 stimulus trials in a random order. There was an additional attention check trial nested within the stimulus trials. Individuals who failed to notice that the attention check trial did not display any temperature data were exited from the survey. The experimental instructions for each condition are provided in the online supplemental materials. The temperature stimuli were generated for Cincinnati and Pittsburgh from means and standard deviations of climate data from NOAA National Centers for Environmental Information (Daily GHNCD data) from 1 March 1999 to 1 March 2013. ${ }^{1}$ Mean temperatures and standard deviations were computed separately for each month and for each city. Each trial consisted of a daily high and low temperature for a random day in the months of January, April, July, and October (to capture all four seasons). The 32 trials were generated by selecting 8 high/low temperatures that ranged between -3 and 3 standard deviations from the monthly mean (see Fig. 2). Detailed information about the temperature stimuli is provided in the online supplemental material.

Next, the participants completed a second short signal detection task to measure their knowledge about the causes of climate change. Table 2 displays a list of actual and bogus causes of climate change. The participants were shown this list of potential causes (in random order) and were asked to categorize each as either a cause or not a cause of GW. Their categorizations on this task were used to estimate a measure of bias and accuracy in their knowledge.

Next, each participant responded to scales measuring their existence beliefs in global warming, beliefs in personal experience with global warming, proenvironmental world views, intentions to act, feelings of response-efficacy of cooperation, and belief in the free market system (Broomell et al. 2015; Heath and Gifford 2006; Bord et al. 2000). The final portion of the experiment asked respondents for their demographic information. A complete list of the stimuli and descriptive statistics is provided in the online supplemental material. We did not measure any additional variables beyond those reported here.

\footnotetext{
${ }^{1}$ The data were collected from NOAA's web page (https://www. ncdc.noaa.gov/cdo-web/search?datasetid=GHCND). We searched by city for Pittsburgh (and Cincinnati) to receive daily temperatures from 1 Mar 1999 to 1 Mar 2013 for each location. For Pittsburgh, daily temperatures were taken from weather station USW00014762. For Cincinnati, daily temperatures were taken from weather station USC00331550.
} 

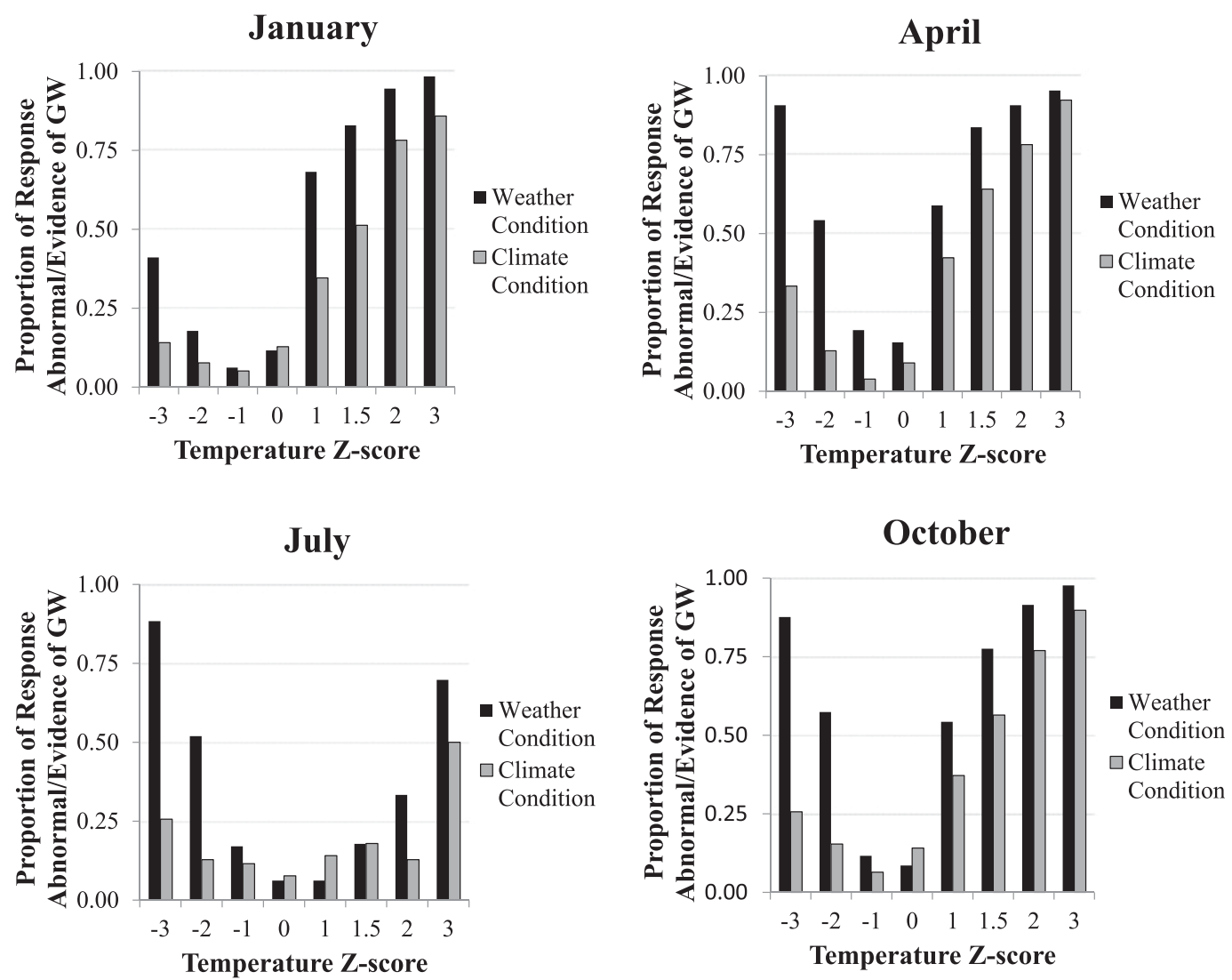

FIG. 2. The proportion of response in the weather condition (choosing abnormality) and the climate condition (choosing evidence of $\mathrm{GW}$ ) as a function of temperature $z$ score separately for each month.

\section{d. Estimating SDT parameters}

Four of our measures were based on estimates of SDT parameters of sensitivity and decision threshold from (a) interpreting temperatures and (b) identifying causes of GW. Estimates of SDT parameters are based on hit rates and false alarm rates. The hit rate is the proportion of trials where the participant responds "signal" when the trial is a true signal. The false alarm rate is the proportion of trials where the participant responds "signal" when the trial is not a true signal. In the context of our experiment, there is no unambiguous true signal. However, we can define trials as representing the signal based on the local warming mental model so that our estimates represent how similar our participant's responses were to this heuristic rule. We therefore interpret our SDT estimates as reflecting the degree to which perceptions of temperatures adhere to the local warming mental model of GW. Our analysis reveals the factors that significantly predict when individuals deviate from this mental model.

To estimate sensitivity and decision threshold for the temperature task, we evaluated the participants' responses using the following definitions for a signal.

\section{1) DeFining ABNORMAL WEATHER}

For the weather condition, we classified temperatures that were 2 (or more) standard deviations above or below the monthly mean as abnormal. This represents large deviations from the monthly mean in temperature. All other trials are classified as normal.

\section{2) DEFINING WEATHER THAT REPRESENT EVIDENCE OF GW}

For the climate condition, we estimated the SDT parameters based on a definition of evidence of $\mathrm{GW}$ that reflects the local warming mental model. We classified daily temperatures that were higher than the monthly mean as representing evidence of GW. This definition is not meant to represent the scientific definition of GW. Additionally, this rule further approximates the GW predictions by focusing only on temperatures that are warmer than the mean, excluding predictions associated with increased variance (where colder temperatures are also to become more frequent). We provided a sensitivity analysis in the online supplemental showing that the overall conclusions do not 
TABLE 2. List of stimuli used to measure knowledge bias and accuracy in causes of global warming. Items in bold indicate systematic inaccuracies in knowledge about the causes of global warming.

\begin{tabular}{|c|c|c|}
\hline & & $\begin{array}{c}\text { Proportion of } \\
\text { participants } \\
\text { responding "cause"" }\end{array}$ \\
\hline \multicolumn{3}{|c|}{ Actual causes } \\
\hline 1 & $\begin{array}{l}\text { Air pollution/emissions from } \\
\text { business and industry. }\end{array}$ & 0.95 \\
\hline 2 & People driving their cars. & 0.88 \\
\hline 3 & $\begin{array}{l}\text { Use of coal and oil by utilities or } \\
\text { electric companies. }\end{array}$ & 0.75 \\
\hline 4 & $\begin{array}{l}\text { People heating and cooling their } \\
\text { homes. }\end{array}$ & 0.57 \\
\hline 5 & Destruction of tropical forests. & 0.91 \\
\hline 6 & $\begin{array}{l}\text { Build of up of greenhouse gases } \\
\text { like } \mathrm{CO}_{2} \text {. }\end{array}$ & 0.89 \\
\hline 7 & $\begin{array}{l}\text { Changes in land usage to farming } \\
\text { and agriculture. }\end{array}$ & 0.53 \\
\hline 8 & Methane produced by livestock. & 0.53 \\
\hline \multicolumn{3}{|c|}{ Bogus causes } \\
\hline 9 & Use of aerosol spray cans. & 0.79 \\
\hline 10 & $\begin{array}{l}\text { Use of chemicals to destroy insect } \\
\text { pests. }\end{array}$ & 0.67 \\
\hline 11 & $\begin{array}{l}\text { Depletion of ozone in the upper } \\
\text { atmosphere. }\end{array}$ & 0.95 \\
\hline 12 & Nuclear power generation. & 0.60 \\
\hline 13 & Overfishing. & 0.21 \\
\hline 14 & $\begin{array}{l}\text { Contamination from hydraulic } \\
\text { fracturing (fracking). }\end{array}$ & 0.56 \\
\hline 15 & $\begin{array}{l}\text { Development and building on the } \\
\text { shore line. }\end{array}$ & 0.41 \\
\hline 16 & $\begin{array}{l}\text { Sulfides in the air from volcanic } \\
\text { eruptions. }\end{array}$ & 0.65 \\
\hline
\end{tabular}

depend on the specific definition of GW used to estimate the SDT parameters.

We estimated the sensitivity and threshold parameters by creating a receiver operating characteristic (ROC) curve for each participant based on their hits (accurate signal responses based on our definitions), false alarms (inaccurate signal responses), and self-reported confidence. We estimated sensitivity as the area under the ROC curve (AUC) and decision threshold as the criterion $C=-0.5\left[\Phi^{-1}(1-\right.$ Hit Rate $)+\Phi^{-1}(1-$ False Alarm Rate $\left.)\right]$, where $\Phi^{-1}$ is the inverse Gaussian cumulative density function (Gescheider 1997). Detailed descriptions of the SDT parameters, statistical assumption checks, and descriptive statistics are provided in the online supplemental material.

\section{Results}

\section{a. Descriptive statistics}

Figure 2 shows responses to the first signal detection task, presented as the proportion of participants who described each temperature as abnormal. Standardized temperatures are presented as their distance, in standard deviations, from the long-term mean for each month and location. In most cases, a higher proportion of participants judged a temperature as "abnormal weather" than as "evidence of GW." Additionally, temperatures with higher proportions of "abnormal" responses also have higher proportions of "evidence of GW" responses.

These data were used to estimate sensitivity and decision threshold. Sensitivity estimates range from 0.5 (random chance performance) to 1.0 (perfect discrimination). Decision threshold estimates range from -3.0 (lax thresholds producing more abnormal/GW responses) to 3.0 (strict thresholds producing more normal responses). The average sensitivity for the weather condition [mean $(M)=0.73$, standard deviation $(\mathrm{SD})=$ 0.09 ] was similar to the climate condition $(M=0.76$, $\mathrm{SD}=0.13$ ), and participants in both conditions performed significantly better than chance in discriminating the temperatures $(p<0.0001)$. The average threshold for the weather condition $(M=-0.12, \mathrm{SD}=0.37)$ was less strict than the climate condition $(M=0.76, \mathrm{SD}=$ 0.81 ), reflecting a higher proportion of participants judging the temperatures as abnormal weather compared to judging the temperatures as evidence of $\mathrm{GW}$.

Table 2 shows responses to the second signal detection task, presented as the proportion of participants indicating each stimulus as a cause of GW. These proportions show that several causes are misunderstood by our sample. The overall average sensitivity for knowledge of causes $(M=0.61, \mathrm{SD}=0.15)$ is lower than that of the temperature task, but still significantly better than chance $(p<0.0001)$. The overall average decision threshold $(M=-0.73, \mathrm{SD}=0.96)$ shows that participants have a lax threshold, meaning they tend to judge these stimuli as true causes.

\section{b. Regression analysis}

Table 3 shows results of regression analyses predicting the sensitivity and threshold in the temperature task. We regressed sensitivity and threshold onto four sets of predictors: (a) experimental condition (weather or climate) and location (Cincinnati or Pittsburgh), (b) demographic variables, (c) the six scales measuring beliefs about GW, and (d) sensitivity and threshold in the task measuring knowledge of causes of GW. The regression model includes an interaction term between experimental condition and the individual-level covariates, allowing for different slopes in each condition. Significant interactions represent differential effects of demographics, beliefs, and knowledge of causes on perceiving abnormality in weather versus perceiving evidence of GW. 
TABLE 3. Coefficient estimates (and standard errors) for predicting decision threshold and sensitivity in classifying temperatures. Values in bold with an asterisk $\left(^{*}\right)$ indicate $p<0.05$. Frame condition is coded as Weather $=0$, Climate $=1$; Location is coded as Cincinnati $=0$, Pittsburgh $=1 ;$ Gender is coded as Male $=0 ;$ Female $=1$. The decision threshold for interpreting temperatures is neutral at 0 , with negative values indicating a lax criterion (bias for using the abnormal/evidence of $\mathrm{GW}$ response) and positive values indicating a strict criterion (bias for using the normal/not evidence of GW response). The decision threshold for identifying causes of GW is neutral at 0 , with negative values indicating a lax criterion (bias for using the "cause" response) and positive values indicating a strict criterion (bias for the "not a cause" response).

\begin{tabular}{|c|c|c|c|c|c|c|c|c|}
\hline \multirow{2}{*}{$\begin{aligned} \text { Predictor } \\
\text { Intercept }\end{aligned}$} & \multicolumn{4}{|c|}{$\begin{array}{l}\text { Decision threshold for temperatures } \\
\text { (criterion } C \text { ) }\end{array}$} & \multicolumn{4}{|c|}{$\begin{array}{l}\text { Sensitivity for temperatures } \\
\text { (area under the ROC curve) }\end{array}$} \\
\hline & \multicolumn{2}{|c|}{$\begin{array}{l}\text { Estimate } \\
-0.11\end{array}$} & \multicolumn{2}{|c|}{$\begin{array}{l}\text { Std. err. } \\
0.11\end{array}$} & \multicolumn{2}{|c|}{$\begin{array}{l}\text { Estimate } \\
\mathbf{0 . 6 9} *\end{array}$} & \multicolumn{2}{|c|}{$\begin{array}{l}\text { Std. err. } \\
0.02\end{array}$} \\
\hline \multicolumn{9}{|l|}{ Design variables } \\
\hline Frame condition & \multicolumn{2}{|c|}{$\mathbf{0 . 7 3} *$} & \multirow{2}{*}{\multicolumn{2}{|c|}{$\begin{array}{l}0.30 \\
0.07\end{array}$}} & \multicolumn{2}{|c|}{$0.16 *$} & \multicolumn{2}{|c|}{0.06} \\
\hline \multirow[t]{3}{*}{ Location } & \multicolumn{2}{|c|}{-0.02} & & & \multicolumn{2}{|c|}{0.02} & \multicolumn{2}{|c|}{0.01} \\
\hline & \multicolumn{2}{|c|}{$\begin{array}{c}\text { Main effect } \\
\text { (weather frame } \\
\text { baseline slope) }\end{array}$} & \multicolumn{2}{|c|}{$\begin{array}{l}\text { Interaction effect (change } \\
\text { in slope for climate frame } \\
\text { relative to weather frame) }\end{array}$} & \multicolumn{2}{|c|}{$\begin{array}{c}\text { Main effect } \\
\text { (weather frame } \\
\text { baseline slope) }\end{array}$} & \multicolumn{2}{|c|}{$\begin{array}{l}\text { Interaction effect (change } \\
\text { in slope for climate frame } \\
\text { relative to weather frame) }\end{array}$} \\
\hline & Est. & S.E. & Est. & S.E. & Est. & S.E. & Est. & S.E. \\
\hline \multicolumn{9}{|l|}{ Demographics } \\
\hline Democrat & 0.06 & 0.11 & -0.31 & 0.18 & 0.02 & 0.02 & -0.02 & 0.04 \\
\hline Republican & 0.00 & 0.12 & -0.22 & 0.20 & -0.01 & 0.03 & -0.01 & 0.04 \\
\hline Age & -0.07 & 0.05 & $0.22 *$ & 0.07 & 0.01 & 0.01 & 0.02 & 0.01 \\
\hline Gender & -0.03 & 0.10 & -0.20 & 0.18 & 0.02 & 0.02 & -0.05 & 0.03 \\
\hline Education & -0.08 & 0.05 & $0.17 *$ & 0.08 & 0.01 & 0.01 & -0.02 & 0.02 \\
\hline \multicolumn{9}{|l|}{ Beliefs about GW } \\
\hline Existence belief & -0.04 & 0.09 & -0.06 & 0.13 & 0.01 & 0.02 & -0.01 & 0.03 \\
\hline Personal experience belief & 0.08 & 0.08 & $-0.44 *$ & 0.11 & -0.01 & 0.02 & 0.03 & 0.02 \\
\hline Environmental worldview & 0.03 & 0.07 & 0.02 & 0.11 & 0.00 & 0.01 & 0.04 & 0.02 \\
\hline Response-efficacy & -0.01 & 0.06 & 0.17 & 0.09 & -0.02 & 0.01 & 0.03 & 0.02 \\
\hline Intention to act & 0.06 & 0.06 & -0.02 & 0.11 & 0.01 & 0.01 & -0.03 & 0.02 \\
\hline Free market system & 0.08 & 0.06 & -0.09 & 0.09 & -0.01 & 0.01 & 0.01 & 0.02 \\
\hline \multicolumn{9}{|l|}{ Knowledge of causes } \\
\hline Sensitivity & -0.03 & 0.05 & 0.07 & 0.08 & $0.03 *$ & 0.01 & 0.01 & 0.02 \\
\hline Threshold & 0.03 & 0.05 & $0.19 *$ & 0.09 & $0.03 *$ & 0.01 & -0.02 & 0.02 \\
\hline$R^{2}$ & & & 0.62 & & & & 0.30 & \\
\hline
\end{tabular}

Table 3 displays the coefficients estimated for sensitivity and threshold separately. The predictors account for less variance in sensitivity $\left(R^{2}=0.30\right)$ than in the decision threshold $\left(R^{2}=0.62\right)$. The top of the table reveals significantly greater sensitivity and a stricter decision threshold in the climate condition, compared to the weather condition. There was no location effect. The bottom of the table shows significant main effects for predicting sensitivity from the "knowledge of causes" task. There were significant interactions between experimental condition and several of the individual-level variables for predicting decision threshold, while there were no interactions for predicting sensitivity. Specifically, there was a significant interaction effect between experimental condition and age, education, personal experiences with GW, and decision threshold for identifying causes as evidence of GW. Figure 3 displays the theoretically relevant interactions between condition assignment and both of the latter variables (personal experience and decision threshold for identifying causes). Individuals reporting beliefs of having experienced GW had a similar decision threshold in the two conditions. However, individuals reporting beliefs of not having experienced $\mathrm{GW}$ had a less strict decision threshold for responding "abnormal" compared to responding "evidence of GW." The pattern of results is similar for decision threshold for identifying causes (see the online supplement for extended results).

\section{Conclusions}

When evaluating the risks of global warming, climate scientists and nonclimate scientists may have different perspectives of what constitutes evidence of GW. Previous research suggests that the general public tends to view their experiences with weather and daily temperatures as evidence of GW, which does not necessarily reflect the large-scale averages that make up the scientific evidence of GW. Even more problematic is the finding that members of the public may have different interpretations of the 

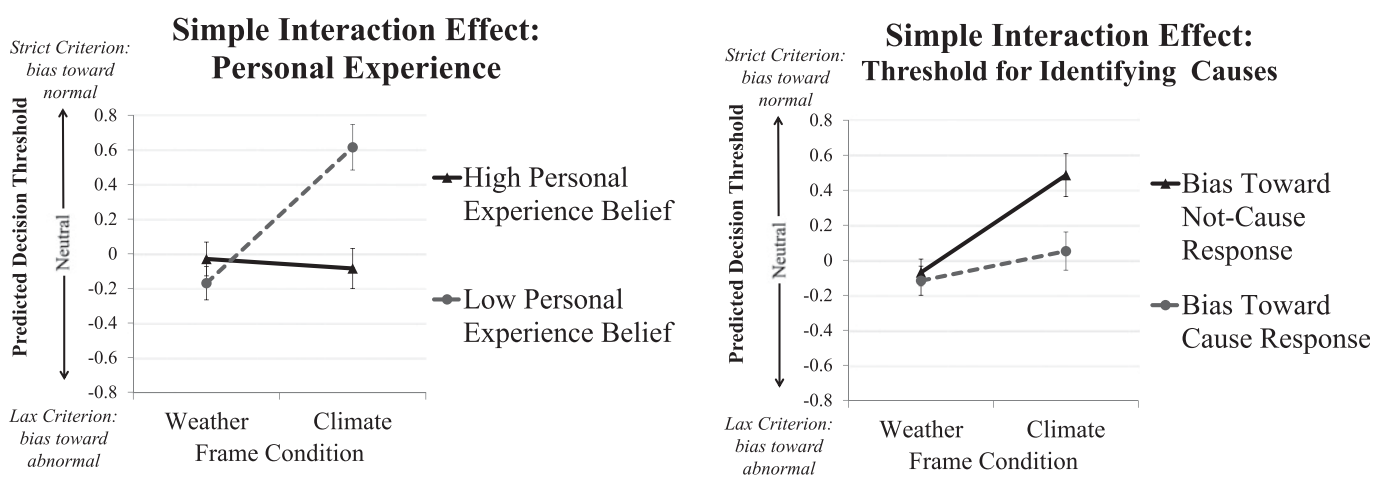

FIG. 3. The simple interaction effect for predicting decision threshold of temperatures based on interactions between the condition assignment and (a) belief in personal experience with GW or (b) the decision threshold for identifying causes. These plots are generated by displaying the predicted mean decision threshold when using values for the predictor variables that are one standard deviation above and below the mean, within each experimental condition.

same experiences in terms of the evidence it provides for GW. In other words, it is not only scientifically inaccurate to point to hot days as evidence of GW, but also a potential barrier to generating stable support for mitigating $\mathrm{GW}$. It is therefore essential to understand the mechanisms that drive differences in interpretations of the same observed temperatures across individuals.

Interpretations of experiences may differ between individuals with different beliefs because of differences in perceptual ability (e.g., belief in GW may lead to paying more attention to typical temperatures) or because of differences in how abnormal a temperature needs to be before it is considered evidence (e.g., belief in GW leads to seeing more temperatures as too warm). Our experimental results highlight the latter as the primary pathway through which motivations distort perceptions of temperatures as evidence of global warming. Specifically, we find that belief-driven motivational distortions for interpreting temperatures as evidence of GW are produced through distortions of the decision threshold (i.e., deciding what constitutes evidence of $\mathrm{GW}$ ), and not through distortions in recognizing normal temperatures (i.e., failing to see the evidence).

Our results suggest that people who do not believe in GW know when it is warmer than average, as evidenced by their similar sensitivity in both conditions; they just do not think that it is evidence of a change in climate, which for an individual day is true. However, in our experiment, all individuals (regardless of belief in $\mathrm{GW}$ ) demonstrated similar ability to sort temperatures that were warmer as evidence of $\mathrm{GW}$, showing that they equally adhere to the local warming mental model. It is therefore unlikely that knowledge about the scientific definition of GW among people who do not believe in $\mathrm{GW}$ is responsible for this decision strategy. These results suggest that individuals who do not believe in global warming have a stricter threshold (i.e., require stronger evidence) for attributing an abnormally warm temperature to GW. Next we discuss the limitations of our experiment and then further discuss and interpret our results for estimates of sensitivity and decision threshold in more detail.

\section{a. Limitations}

The goal of our study was to explore the mechanisms through which people interpret the same information differently. Some of these differences are theorized to be caused by personal beliefs and ideology, which present many obstacles to their study. First, beliefs and ideology are individual characteristics that cannot be randomly assigned. Through traditional experimentation, we can only gain correlational knowledge about the relationship between beliefs and interpretations in the weather. We are thus limited in the inferences we can make based on the evidence presented in this study. This evidence is useful for predicting that, when judging climate change based on weather, people with different beliefs will tend to be associated with predictably different interpretations. Likewise, we can predict the absence of this association for perceptions of abnormal weather. However, we cannot interpret our evidence as suggesting that intervening on beliefs will cause the same effect on interpretations of temperatures. Second, while highly unlikely, we cannot definitely rule out the possibility that when subjects participated in categorizing our temperature stimuli, their performance on this task changed our measurement of their beliefs. We provide extended analyses in the online supplemental material showing that our results are robust to this type of effect. 
One final limitation of our study is related to our constrained geographical sampling. Temperature variation, seasons, and average temperatures differ depending on geography. Our sample is limited to two local cities within the United States that have similar seasons and average temperatures. However, given the availability of temperature data from NOAA, there is no reason that this experiment could not be replicated in other locations in the United States. Our limited representation opens the possibility that these results could change in other locations, for example, that (a) never frost or (b) have dry and wet seasons. Within the constraints of these limitations, we next provide further discussion of our experimental results.

\section{b. Sensitivity}

Our estimates of sensitivity add a new dimension to previous findings. They show that individual differences in sensitivity to identifying temperatures warmer than average do not contribute to the previous findings that beliefs influence perceptions of personal experience with GW. Our estimates of sensitivity show that participants performed significantly above chance level on the temperature task when framed as climate, suggesting that our sample was indeed ready and able to evaluate GW in terms of local warming. This result supports previous research that the general public tends to believe in the local warming model ( $\mathrm{Li}$ et al. 2011). It also takes this research a step further, finding that individuals with different beliefs tend to interpret daily temperatures with the same level of sensitivity.

Sensitivity is related to the criteria we used to evaluate the participants' responses. We did not tell participants that we defined abnormal temperatures as 2 or more standard deviations from the mean. We intentionally withheld this information because we were interested to see our participants' natural interpretation of abnormality in temperatures. However, future experiments of this type could also look at sensitivity in terms of the ability to apply an externally imposed rule for identifying abnormality. For example, future research might measure performance on the task of identifying temperatures that locally occur less than $1 \%$ of the time or the task of identifying temperatures that are warmer than average. This type of approach has the added benefit of having an objective definition of hits and false alarms because the participants are directly provided the true criteria for measuring detection performance.

\section{c. Decision thresholds}

Our analysis of decision thresholds for interpreting daily temperatures informs on our research questions: Are people who believe in GW seeing evidence in most temperatures? Are people who do not believe in GW not seeing evidence in most temperatures? We further interpret the implications of the fitted mean differences across the framing conditions in our estimates of decision threshold for those who believe in GW and those who do not believe in GW (see Fig. 3). However, the interpretation depends on the modeling assumptions. We assumed that warmer than average temperatures represent $\mathrm{GW}$ in the local warming mental model (as discussed in section 2). If our sample relied on the local warming mental model we would see the following results: (a) participants who report more belief in experiencing GW display the same threshold across conditions and (b) participants who reported less belief in experiencing GW display a stricter threshold in the climate condition compared to the weather condition. This pattern suggests that those who do not believe in GW apply a stricter threshold to what would constitute evidence of GW relative to what would constitute abnormal weather for the same set of potential temperatures. In other words, individuals who do not believe they have experienced GW tend to only believe that extremely warm temperatures are evidence of $\mathrm{GW}$, failing to accept less extreme-but still warmer than averagetemperatures as evidence of GW.

As previously mentioned, changing our modeling assumptions can change this type of interpretation of the data. It is possible to have a different definition for which temperatures represent signal and noise, for example, that none of the temperatures are evidence of GW because local temperatures are not truly indicative of global averages. We can check the robustness of our result by evaluating the estimates of decision threshold under this different signal detection model (see the online supplemental material). Assuming that our participants applied this alternative rule (which is not supported by our data; see Fig. 2), it would reverse the direction in the interpretation of the data to the following results: (a) participants who report more belief in experiencing GW display a more lenient threshold in the climate condition compared to the weather condition and (b) participants who reported less belief in experiencing GW display the same threshold across conditions. This pattern suggests that those who do believe in GW apply a lenient threshold that produces more evidence of GW relative to what would constitute abnormal weather for the same set of potential temperatures. Either way, these results support a motivational distortion of judgment through decision thresholds. A more detailed discussion of the robustness of this result is provided in the online supplemental material.

These results also have practical implications for communicating about the impacts of GW. Ideally, 
communications would provide a proper long-term perspective of GW as global averages and emphasize the unreliability of local weather for providing information about global trends. However, given the strong tendency to overinterpret evidence from small samples (Tversky and Kahneman 1971), it would be unreasonable to expect any single GW communication to address such fundamental issues of intuitive statistical inference. Given that our results isolate decision thresholds as a fundamental source of polarization, a more feasible communication strategy may be to convey appropriate decision thresholds. Given the inevitability of people thinking about daily temperatures, they might only be noted in GW communications when they have repeatedly beaten previous records, along with appropriate qualifiers. Additionally, appropriate thresholds linking weather to GW can be defined by scientific expertise, and tallies of the frequency of extreme weather events breaking these thresholds can be made publically available across different localities. While this approach is informed by our results, our experiment has limited geographic representation, so any such communication strategies should be empirically tested on their target audience before implementation.

Given the many pitfalls in linking observed weather to evidence for GW, it might be best to avoid communication regarding weather altogether. There is (a) difficulty in putting extremely cold weather in perspective, (b) large amounts of variability in weather across seasons, years, and geographic locations that mask the signal in noise, and (c) the tendency for experiences to distort our perceptions of probability (Weber 2013). A better investment might be focusing on long-term impacts that cannot be construed as personally observable (e.g., ocean acidification) to reduce the temptation to attempt to verify scientific evidence through personal observation. As ever, communications must be faithful to climate science, reflect psychology of judgment, reflect the needs of the public, and undergo empirical testing.

Acknowledgments. We thank Jessica Broomell and Baruch Fischhoff for comments on a previous draft. We also thank Kelly Klima and Iris Grossmann for their valuable advice on the climate science aspects of our experiments.

\section{REFERENCES}

Bord, R. J., R. E. O'Connor, and A. Fisher, 2000: In what sense does the public need to understand global climate change? Public Understanding Sci., 9, 205-218, doi:10.1088/ 0963-6625/9/3/301.
Broomell, S. B., D. V. Budescu, and H. H. Por, 2015: Personal experience with climate change predicts intentions to act. Global Environ. Change, 32, 67-73, doi:10.1016/j.gloenvcha.2015.03.001.

Budescu, D. V., H. H. Por, and S. B. Broomell, 2012: Effective communication of uncertainty in the IPCC reports. Climatic Change, 113, 181-200, doi:10.1007/s10584-011-0330-3.

Dewitt, B., B. Fischhoff, A. Davis, and S. B. Broomell, 2015: Environmental risk perception from visual cues: Caution and sensitivity in evaluating tornado risks. Environ. Res. Lett., 10, 124009, doi:10.1088/1748-9326/10/12/124009.

Farris, C., T. A. Treat, R. J. Viken, and R. M. McFall, 2008a: Sexual coercion and the misperception of sexual intent. Clin. Psychol. Rev., 28, 48-66, doi:10.1016/j.cpr.2007.03.002.

,,--- , and,$- 2008 \mathrm{~b}$ : Perceptual mechanisms that characterize gender differences in decoding women's sexual intent. Psychol. Sci., 19, 348-354, doi:10.1111/ j.1467-9280.2008.02092.x.

Gescheider, G., 1997: Psychophysics: The Fundamentals. 3rd ed. Lawrence Erlbaum Associates, 435 pp.

Goebbert, K., H. C. Jenkins-Smith, K. Klockow, M. C. Nowlin, and C. L. Silva, 2012: Weather, climate, and worldviews: The sources and consequences of public perceptions of changes in local weather patterns. Wea. Climate Soc., 4, 132-144, doi:10.1175/WCAS-D-11-00044.1.

Green, D. M., and J. A. Swets, 1988: Signal Detection Theory and Psychophysics. Peninsula Publishing, 521 pp.

Heath, Y., and R. Gifford, 2006: Free-market ideology and environmental degradation: The case of belief in global climate change. Environ. Behav., 38, 48-71, doi:10.1177/ 0013916505277998.

Howe, P. D., and A. Leiserowitz, 2013: Who remembers a hot summer or a cold winter? The asymmetric effect of beliefs about global warming on perceptions of local climate conditions in the U.S. Global Environ. Change, 23, 1488-1500, doi:10.1016/j.gloenvcha.2013.09.014.

IPCC, 2014: Climate Change 2014: Synthesis Report. R. K. Pachauri and L. A. Meyer, Eds., IPCC, 151 pp.

Kahan, D. M., E. Peters, M. Wittlin, P. Slovic, L. L. Ouellette, D. Braman, and G. Mandel, 2012: The polarizing impact of science literacy and numeracy on perceived climate change risks. Nat. Climate Change, 2, 732-735, doi:10.1038/nclimate1547.

Kahneman, D., and Frederick, S., 2002: Representativeness revisited: Attribute substitution in intuitive judgment. Heuristics and Biases, T. Gilovich, D. Griffin, and D. Kahneman, Eds., Cambridge University Press, 49-81.

Kunda, Z., 1990: The case for motivated reasoning. Psychol. Bull., 108, 480-498, doi:10.1037/0033-2909.108.3.480.

Lewandowsky, S., G. E. Gignac, and K. Oberauer, 2013: The role of conspiracist ideation and worldviews in predicting rejection of science. PLoS One, 8, e75637, doi:10.1371/ journal.pone.0075637.

Li, Y., E. J. Johnson, and L. Zaval, 2011: Local warming: Daily temperature change influences belief in global warming. Psychol. Sci., 22, 454-459, doi:10.1177/0956797611400913.

Macmillan, N. A., and C. D. Creelman, 2004: Detection Theory: A User's Guide. Psychology Press, 512 pp.

McCright, A. M., and R. E. Dunlap, 2011: Cool dudes: The denial of climate change among conservative white males in the United States. Global Environ. Change, 21, 1163-1172, doi:10.1016/j.gloenvcha.2011.06.003.

, - — and C. Xiao, 2014: The impacts of temperature anomalies and political orientation on perceived winter warming. Nat. Climate Change, 4, 1077-1081, doi:10.1038/nclimate2443. 
Melillo, J. M., T. C. Richmond, and G. W. Yohe, 2014: Highlights of Climate Change Impacts in the United States: The Third National Climate Assessment. U.S. Global Change Research Program, $148 \mathrm{pp}$.

Myers, T. A., E. W. Maibach, C. Roser-Renouf, K. Akerlof, and A. A. Leiserowitz, 2013: The relationship between personal experience and belief in the reality of global warming. Nat. Climate Change, 3, 343-347, doi:10.1038/nclimate1754.

Nickerson, R. S., 1998: Confirmation bias: A ubiquitous phenomenon in many guises. Rev. Gen. Psychol., 2, 175-220, doi:10.1037/1089-2680.2.2.175.

Risen, J. L., and C. R. Critcher, 2011: Visceral fit: While in a visceral state, associated states of the world seem more likely. J. Pers. Soc. Psychol., 100, 777-793, doi:10.1037/ a0022460.

Spence, A., W. Poortinga, C. Butler, and N. F. Pidgeon, 2011: Perceptions of climate change and willingness to save energy related to flood experience. Nat. Climate Change, 1, 46-49, doi:10.1038/nclimate1059.
Trenberth, K. E., 2012: Framing the way to relate climate extremes to climate change. Climatic Change, 115, 283-290, doi:10.1007/ s10584-012-0441-5.

Tversky, A., and D. Kahneman, 1971: Belief in the law of small numbers. Psychol. Bull., 76, 105-110, doi:10.1037/h0031322.

Weber, E. U., 2006: Experience-based and description-based perceptions of long-term risk: Why global warming does not scare us (yet). Climatic Change, 77, 103-120, doi:10.1007/ s10584-006-9060-3.

- 2013: Psychology: Seeing is believing. Nat. Climate Change, $\mathbf{3}$, 312-313, doi:10.1038/nclimate1859.

— in the United States. Amer. Psychol., 66, 315-328, doi:10.1037/ a0023253.

Wickens, T. D., 2001: Elementary Signal Detection Theory. Oxford University Press, 262 pp.

Zaval, L., E. A. Keenan, E. J. Johnson, and E. U. Weber, 2014: How warm days increase belief in global warming. Nat. Climate Change, 4, 143-147, doi:10.1038/nclimate2093. 